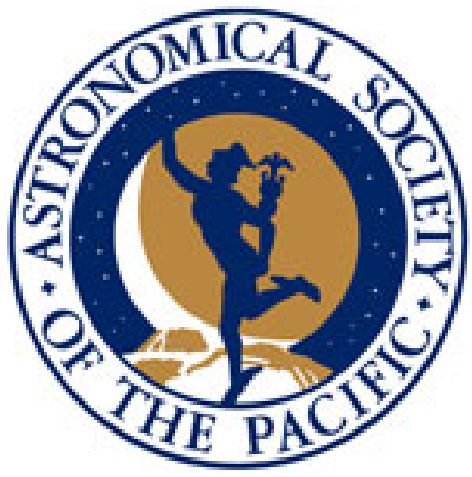

An Optical Pointing Telescope for Radio Astronomy

Author(s): Sidhi Assawaworrarit and Stephen Padin

Reviewed work(s):

Source: Publications of the Astronomical Society of the Pacific, Vol. 124, No. 913 (March 2012), pp. 242-246

Published by: The University of Chicago Press on behalf of the Astronomical Society of the Pacific

Stable URL: http://www.jstor.org/stable/10.1086/664969

Accessed: $2 7 \longdiv { / 0 4 / 2 0 1 2 1 1 : 4 8 }$

Your use of the JSTOR archive indicates your acceptance of the Terms \& Conditions of Use, available at http://www.jstor.org/page/info/about/policies/terms.jsp

JSTOR is a not-for-profit service that helps scholars, researchers, and students discover, use, and build upon a wide range of content in a trusted digital archive. We use information technology and tools to increase productivity and facilitate new forms of scholarship. For more information about JSTOR, please contact support@ jstor.org. 


\title{
An Optical Pointing Telescope for Radio Astronomy
}

\author{
SidHi ASSAWAWORRarit AND STEPHEN PAdin \\ California Institute of Technology, MC 367-17, Pasadena, CA 91125; sassawaw@caltech.edu, \\ spadin@caltech.edu \\ Received 2012 January 9; accepted 2012 January 31; published 2012 February 21
}

\begin{abstract}
Design details are given for a stable optical pointing telescope for radio astronomy. The telescope is a $100 \mathrm{~mm} \mathrm{f} / 15$ refractor with the objective glued to a ring of three blade flexures, an insulated and vented Invar tube mounted on flexures, and an axially symmetric camera mount. For a pair of identical telescopes, the rms differential pointing stability is $0.1^{\prime \prime} \mathrm{hr}^{-1}$ over $2 \mathrm{hr}, 0.05^{\prime \prime}$ day $^{-1}$ over 3 days, $0.03^{\prime \prime} \mathrm{K}^{-1}$, and $0.1^{\prime \prime}$ after a $90^{\circ}$ change in elevation.
\end{abstract}

\section{INTRODUCTION}

Radio telescopes are often equipped with a small optical telescope that is used to observe stars in order to measure the pointing model (Mangum et al. 2006; Carlstrom et al. 2011). This approach is generally faster than radio pointing measurements, and it yields the tilt and offset of each telescope axis, leaving just the flexure and radio-to-optical collimation terms to be obtained from radio measurements. Optical measurements are useful for checking the pointing performance, and for identifying servo problems, so they play an important role in the commissioning of a radio telescope. A small optical telescope can also be used for offset guiding at night, if the field of view is large enough.

Small optical telescopes are hardly a new technology, but the design constraints for a pointing telescope are unusual in that they emphasize stability over image quality. A pointing telescope must be stable during a pointing model measurement, which might take $1 / 2 \mathrm{hr}$ with $1 \mathrm{~K}$ temperature variations. Stability on longer timescales is useful for following changes in the pointing model.

The work described here was motivated by CCAT, a $25 \mathrm{~m}$ diameter submillimeter telescope that will be located on Cerro Chajnantor in the Atacama Desert. CCAT will have a 3.5" beam at $\lambda=350 \mu \mathrm{m}$, an offset pointing requirement of $1 / 10$ beamwidth within $1^{\circ}$ and $1 \mathrm{hr}$ of the last pointing measurement, and a blind pointing requirement of $1 / 2$ beamwidth. In order to measure the pointing performance at this level, the pointing telescope must be stable to $\sim 0.1^{\prime \prime} \mathrm{hr}^{-1}$ and $\sim 0.1^{\prime \prime} \mathrm{K}^{-1}$. Stability at the $1^{\prime \prime}$ level is straightforward, but $0.1^{\prime \prime}$ is challenging. In the following sections, we describe a pointing telescope that achieves $0.1^{\prime \prime}$ stability. The material is organized as follows. In $\S 2$ we review the key design considerations, describe the telescope optical and mechanical details, and give estimates of the thermal performance. In $\S 3$ we describe measurements of the optical pointing telescope stability.

\section{POINTING TELESCOPE DESIGN}

\subsection{Design Considerations}

Reflectors, refractors, and folded refractors have all been used for pointing telescopes; e.g., the Caltech Submillimeter Observatory (CSO) has a $150 \mathrm{~mm}$ reflector, the Combined Array for Research in Millimeter-wave Astronomy (CARMA) telescopes have a $100 \mathrm{~mm}$ folded refractor (Leighton 1978), and the Atacama Large Millimeter Array (ALMA) telescopes have a $100 \mathrm{~mm}$ refractor with a Barlow lens (Mangum 2000). Refractors tend to be small and lightweight, but designs with just a simple achromat require a fairly long focal length to achieve a reasonable field of view. This leads to a long tube, or a folded design. Refractors are insensitive to a tilt of the objective, but sensitive to a decenter. Reflectors are usually larger, more sensitive, and heavier, but generally shorter because they are folded. A reflector with a flat secondary has little sensitivity to decenter, but reflection from the secondary doubles the effect of a primary tilt. The choice between a reflector and a refractor is not obvious, but the simplicity of a refractor is an important advantage.

The major design issues for a pointing telescope are as follows:

1. Repeatable gravitational deflection. Repeatability is generally better for smaller deflections, so lighter, stiffer designs are preferred. Minimizing the overall mass of the pointing telescope helps reduce deflection of the mount on the radio telescope. Cantilever mounts introduce orientation-dependent deflections, so it is better to maintain axial symmetry in the design.

2. Small, repeatable thermal deformation. Thermal deformation can be minimized by making all the mechanical parts from the same low-coefficient-of-thermal-expansion material, insulating to reduce thermal gradients, and minimizing the power dissipation of the camera. Axial symmetry also helps to reduce thermal deformation. Friction connections between different materials may slip during thermal cycling, so flexure connections 
TABLE 1

TELESCOPE MASS

\begin{tabular}{|c|c|}
\hline Component & $\begin{array}{c}\text { Mass } \\
(\mathrm{kg})\end{array}$ \\
\hline Objective .. & 0.38 \\
\hline Flexures $\ldots$ & 0.11 \\
\hline Tube $\ldots \ldots$. & 3.98 \\
\hline Camera plate & 0.30 \\
\hline Split clamps & 1.26 \\
\hline Total & 6.03 \\
\hline
\end{tabular}

are preferred. Lens and mirror mounts are difficult because of the metal-to-glass connection, so a simple design with fewer optical components is better.

3. Small refractive index variations. Thermal gradients in the air inside and in front of the tube are troublesome because they cause pointing errors that vary with time and tube orientation. Gradients inside the tube can be eliminated by blowing air through the tube, insulating the inside of the tube to reduce heating of the air, and minimizing the power dissipation of the camera. Gradients in front of the tube can be reduced by keeping the optics and tube at ambient temperature. Atmospheric seeing must be handled by making enough measurements on each star to ensure that seeing does not limit the pointing measurement.

\subsection{Design Details}

For CCAT, we chose to prototype a simple optical refractor pointing telescope. The design is based on a commercially available $100 \mathrm{~mm}$ f/15 achromat (Edmund NT50-107) with a diffraction-limited field of view of $1.2^{\circ}$. Figure 1 shows the mechanical details and Table 1 gives masses for the major components. All the metal parts are made of Invar, so the only material transitions are at the lens and camera mounts and where the tube attaches to the radio telescope. The lens and tube attachments are both flexure connections. The tube is $127 \mathrm{~mm}$ diameter $\times 0.75 \mathrm{~mm}$ wall Invar, rolled and riveted. Future versions of the pointing telescope will probably have a welded tube for better long-term stability. The tube is insulated on the inside with $12 \mathrm{~mm}$ thick black polyethylene foam, which also serves as a light baffle, and on the outside with $6 \mathrm{~mm}$ thick aluminized bubble wrap. The objective

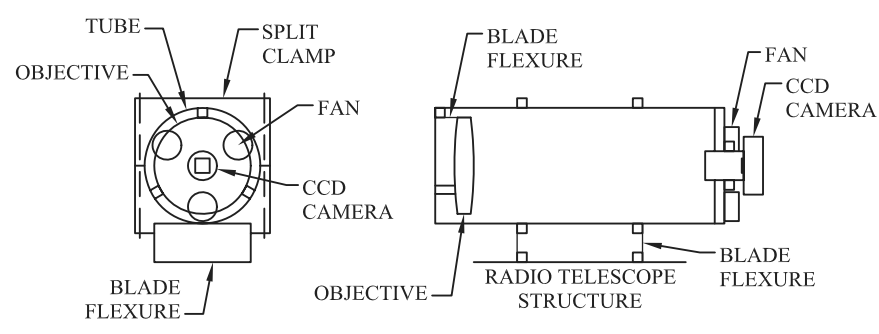

FIG. 1.-Telescope mechanical details.

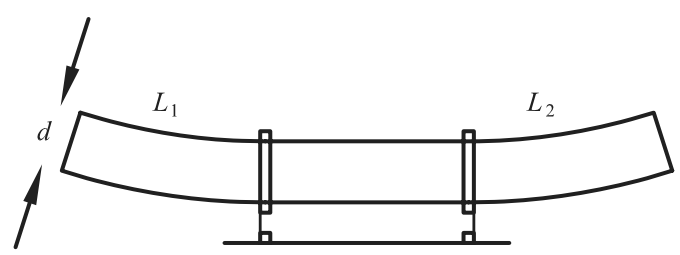

FIG. 2.-Deformation due to a temperature gradient across the tube.

is supported by three equally spaced $20 \mathrm{~mm} \times 75 \mathrm{~mm} \times$ $0.5 \mathrm{~mm}$ Invar blade flexures (Yoder 2006) glued to the rim of the glass with epoxy (Stycast 2850FT). A $12 \mathrm{~mm}$ gap between the objective and the tube allows air to flow through the tube. The flow is driven by three small fans that draw in outside air at the camera end of the tube. The camera has a 1.25 inch diameter aluminum draw tube clamped to a $6 \mathrm{~mm}$ Invar plate mounted in the end of the telescope tube. Future versions of the camera mount will probably have an Invar draw tube glued directly to the CCD facesheet. The telescope tube is supported by a pair of split clamps held in place with long steel bolts (represented by dashed lines in Fig. 1). The clamps are tightened with no gap to prevent the tube from rotating as a result of thermal cycling.

CCAT will be inside an enclosure, so the pointing telescope will not need protection from the weather. For exposed locations, a camera box and a motor-driven lens cap could be added to keep snow out during storms, but the tube would still be vented during pointing observations.

\subsection{Thermal Performance}

In this section, we estimate the effect of thermal deformation and refractive index variations on the performance of the pointing telescope. These estimates illustrate why the telescope must be insulated and vented. A temperature gradient across the tube

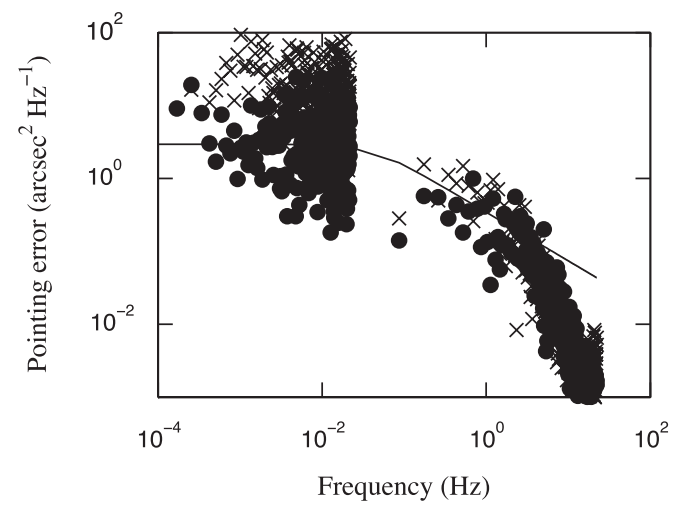

FIG. 3.-Power spectral density of differential pointing errors in azimuth (filled circles) and elevation (crosses) observing a street lamp $\sim 20 \mathrm{~km}$ away. The curve is a von Kármán spectrum $P(f)=A f_{0}^{2 / 3} /\left(f^{2}+f_{0}^{2}\right)^{1 / 3}$, with $A=$ $3 \operatorname{arcsec}^{2} \mathrm{~Hz}^{-1}$ and $f_{0}=0.04 \mathrm{~Hz}$. The low-frequency points are from $\sim 20 \mathrm{~s}$ blocks of stacked images. The high-frequency points are from unstacked images at $\sim 30 \mathrm{~Hz}$ frame rate. 


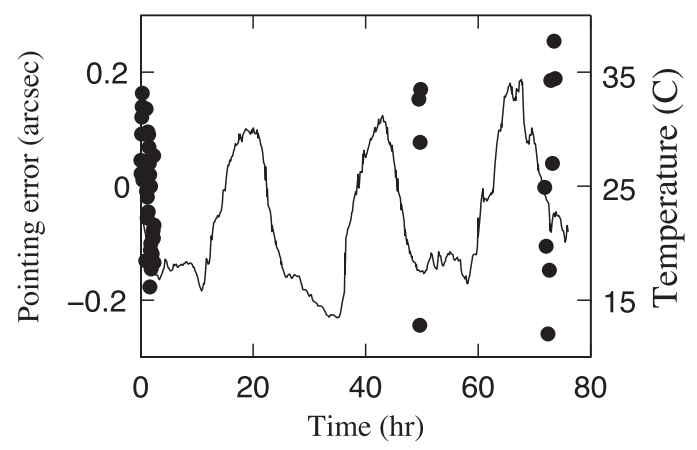

FIG. 4.-Differential pointing error (filled circles) and air temperature (solid curve) vs. time. Start time for the measurement is 2011-September-20 02:49 UT. The error on each point is $\sim 0.07^{\prime \prime}$, based on the scatter of the 25 measurements per point on the first day. The weather was slightly hazy for the second and third days, so the integration time was increased, but the scatter is still a little larger.

lengthens the warmer side of the tube, resulting in a decenter of the optics, as shown in Figure 2. The decenter is

$$
\delta_{i}=\frac{L_{i}^{2} \alpha \Delta T}{2 d}
$$

where $i=\{1,2\}$ indicates the objective or camera, $L_{i}$ is the distance from the tube clamp, $\alpha$ is the coefficient of thermal expansion of the tube, $\Delta T$ is the temperature gradient, and $d$ is the diameter of the tube. The pointing error is

$$
\theta_{T} \approx\left(\delta_{1}-\delta_{2}\right) / f
$$

where $f$ is the focal length. For the CCAT pointing telescopes, $L_{1}=L_{2}=f / 3$, so a uniform gradient causes the same decenter at the objective and camera and there is no pointing error. With $f=1.5 \mathrm{~m}, \alpha=1.2 \times 10^{-6} \mathrm{~K}^{-1}$ (for Invar), $d=127 \mathrm{~mm}$, and $\Delta T=1 \mathrm{~K}$, the worst case of opposite gradients in the two ends of the tube causes a pointing error of $0.3^{\prime \prime}$. Temperature gradients of $1 \mathrm{~K}$ are typical in radio telescope structures, so the pointing telescope tube should be insulated to ensure that pointing errors due to thermal gradients are less than $0.1^{\prime \prime}$.

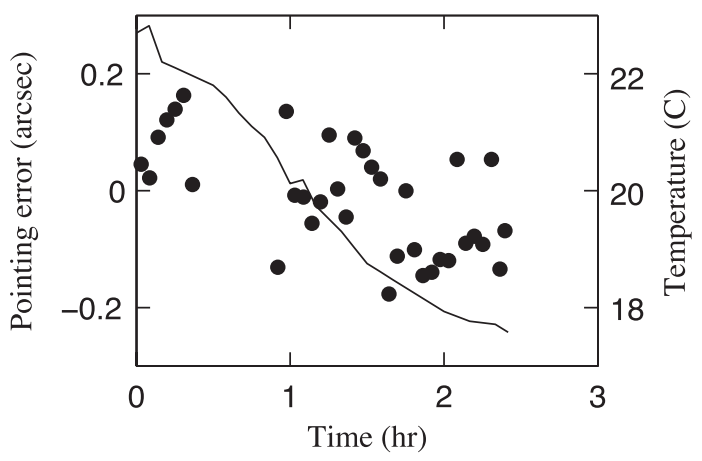

FIG. 5.-Detail of the first day's measurements in Fig. 4.

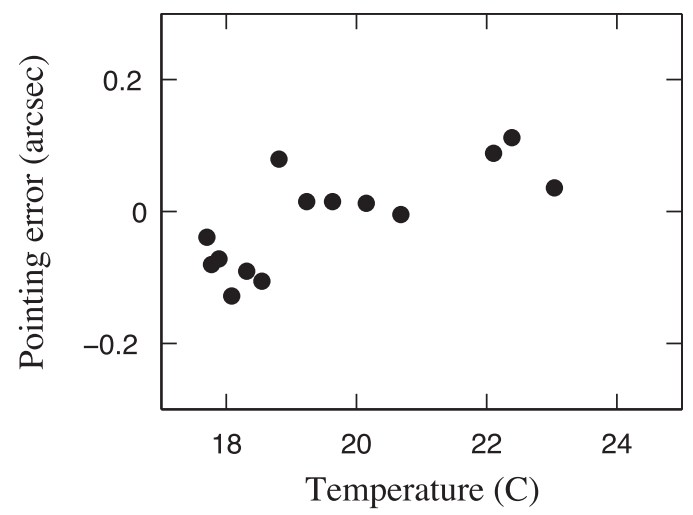

FIG. 6.-Differential pointing error vs. temperature. The error on each point is $\sim 0.05^{\prime \prime}$. These are the same data as in Fig. 4, but binned in temperature.

A thermal gradient across the tube generates a gradient in refractive index, which in turn causes a pointing error. For air, the change in refractive index is (Edlen 1966)

$$
\Delta n \approx-10^{-6} \Delta T,
$$

where $\Delta T$ is in kelvins. The pointing error contribution from a slice of tube of length $\delta l$ at a distance $l$ from the end is

$$
\delta \theta_{n} \approx\left(\frac{\Delta n \delta l}{D}\right) l / f
$$

where $D$ is the diameter of the objective, and the term in parentheses is the tilt of the wavefront leaving the slice. Integrating along the tube gives the total pointing error:

$$
\theta_{n} \approx \frac{\Delta n}{D f} \int_{0}^{f}(f-l) d l=\frac{f \Delta n}{2 D}
$$

With $f=1.5 \mathrm{~m}, D=100 \mathrm{~mm}$, and $\Delta T=1 \mathrm{~K}$, the pointing

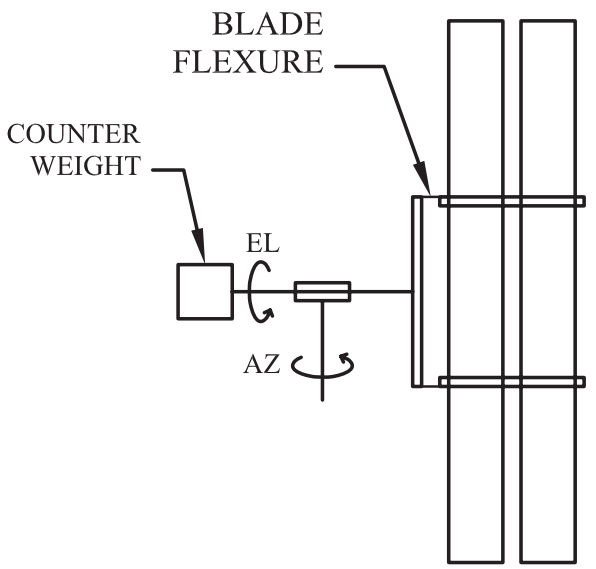

FIG. 7.-Test setup for measuring repeatability of gravitational deformations. 
error is $1.5^{\prime \prime}$. To achieve less than $0.1^{\prime \prime}$ stability, the tube must be vented to suppress refractive index gradients. Insulating the inside of the tube also helps because it reduces heating of the air (and thermal deformation of the tube).

\section{TESTS}

\subsection{Test Setup}

It is difficult to measure the absolute stability of a pointing telescope, so we built two identical telescopes in order to measure their relative performance. Relative measurements are useful because they reveal nonrepeatable errors. Repeatable errors can be calibrated, so they are not as important.

For stability tests, stars and distant street lights were used as sources. The telescopes were equipped with inexpensive board-level CCD cameras (Sentech STC-TB33USB) and AAthermocouple temperature sensors. Images and temperatures were acquired using MATLAB. The CCD sensors (Sony ICX424AL) have $640 \times 4807.4 \mu \mathrm{m}^{2}$ pixels, so the plate scale is $1^{\prime \prime}$ pixel $^{-1}$. Point-source images are typically a few pixels in diameter, which is enough to measure the centroid to a small fraction of a pixel. To minimize the effect of seeing and motion of the source and telescopes, images were taken simultaneously from each telescope. Blocks of images at $\sim 30 \mathrm{~Hz}$ frame rate were then stacked and recorded to disk. A block size of 600 images was used for observations of fixed street lights, but this was reduced to 50 for observations of stars, because they drift slowly in the field. During analysis, a threshold was applied to the stacked images to move zero brightness to 40 or $60 \%$ of the peak signal. The image centroid was then calculated in a $20 \times$ 20 pixel window roughly centered on the source. The threshold was set at $60 \%$ for observations of street lights, where the background was fairly bright, and $40 \%$ for observations of stars.

\subsection{Measurements with Fixed Telescopes}

The thermal and temporal stability of the pointing telescopes was measured with the telescopes fixed on the roof, observing street lights $\sim 20 \mathrm{~km}$ away. The telescope tube clamps were bolted together in a vertical stack and attached to an aluminum support frame with blade flexures. Since the measurements were made near the horizon in Los Angeles, the seeing was poor. Figure 3 shows the power spectrum of differential pointing errors between the two telescopes. Only about half of the air path was common to both telescopes, so the differential seeing was also poor. The absolute seeing at midelevation at a good site is typically a factor of 3 smaller than the differential seeing measured here (Ukita et al. 2008). Based on Figure 3, measurements were averaged for $5 \mathrm{~min}$ in order to obtain the differential pointing to $0.1^{\prime \prime}$. Variations in the vertical temperature gradient made the vertical component of seeing much worse, so only the horizontal component of the differential pointing was measured using this setup. The differential pointing measurements were corrected for relative rotation, tip, and tilt of the cameras,

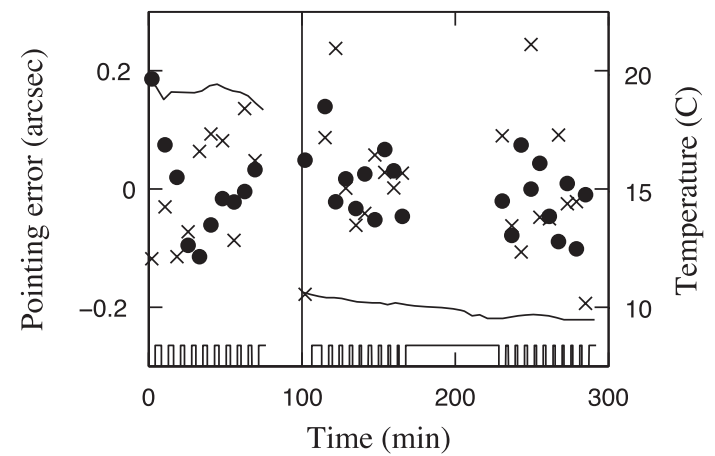

FIG. 8.-Differential pointing error in elevation (filled circles), azimuth (crosses), and air temperature (solid curve) vs. time for two measurements of the repeatability of gravitational deformations. Start times for the two measurements are 2011-November-28 02:10 UT (time $=0$ in the plot) and 2011December-21 02:39 UT (time $=100$ minutes in the plot). The average differential pointing error has been subtracted from each measurement. The error on each point is $\sim 0.1^{\prime \prime}$, based on the scatter of the 50 measurements per point. The waveform along the bottom of the plot indicates elevation vs. time, switching from $34^{\circ}$ during a pointing measurement to $34^{\circ}+90^{\circ}$ between measurements.

obtained from measurements of three street lights at different positions in the field.

Figures 4-6 show the differential pointing error versus time and temperature over 3 days. The telescopes were left on the roof, exposed to high daytime temperatures, but measurements were taken for just a few hours each evening. The rms stability from Figures $4-6$ is $0.1^{\prime \prime} \mathrm{hr}^{-1}$ over $2 \mathrm{hr}, 0.05^{\prime \prime}$ day $^{-1}$ over 3 days, and $0.03^{\prime \prime} \mathrm{K}^{-1}$.

\subsection{Measurements with Moving Telescopes}

The repeatability of differential gravitational deformations was measured with the telescopes stacked side by side on a moving mount. The telescope tube clamps were bolted together, and the assembly was attached with blade flexures to an aluminum plate on the mount, as shown in Figure 7. We repeatedly observed Polaris and then moved the telescopes through an elevation angle of $90^{\circ}$. Polaris was used as the source to give reasonable seeing, but we could have used a street light and a longer averaging time. The relative rotation, tip, and tilt of the cameras were obtained from five observations of Polaris at different field offsets. Figure 8 shows the differential pointing error versus time for two measurements. The rms repeatability from Figure 8 is $0.07 "$ in elevation and $0.1^{\prime \prime}$ in azimuth.

\subsection{Sensitivity and Camera Selection}

The pointing telescope gives saturated images of the brighter Galilean satellites, which have $m_{V} \sim 5$, in a few seconds. At the south Galactic pole, where the density of stars is lowest, the Two Micron All Sky Survey ${ }^{1}$ (2MASS) contains roughly one

\footnotetext{
${ }^{1}$ See http://irsa.ipac.caltech.edu/applications/Stats.
} 
$m_{V}<8$ star per square degree. The field of view of the pointing telescope is $\sim 1^{\circ}$, so with a large enough camera we should always see a saturated star image in a few tens of seconds. This is reasonable for slow, offset guiding when tracking at sidereal rate: e.g., for radio spectroscopy. Changing to a cooled camera would improve the sensitivity, allowing a smaller field of view for guiding. In this case, the power dissipation of the camera would be higher, so thermal deformation of the tube and heating of the air inside the tube could limit the stability of the pointing telescope. It is not clear if offset optical guiding will improve the radio pointing performance, because the thermal variations that offset guiding can correct also cause varying radio-to-optical offsets.

The camera used for testing is only advertised for operation to $0^{\circ} \mathrm{C}$, but the temperature at the CCAT site can be $-20^{\circ} \mathrm{C}$. The CCD sensor can operate well below $0^{\circ} \mathrm{C}$, and cooled cameras using the same sensor are available commercially. Our experience with similar CCD cameras is that they generally work at $-20^{\circ} \mathrm{C}$. If future versions of the pointing telescope require a different camera, the mass, power dissipation, and temperature rise of the camera must be kept small to maintain the stability of the pointing telescope. The mass of the camera used for testing is only $150 \mathrm{~g}$ and its power dissipation is $2 \mathrm{~W}$.

\subsection{Reflector Tests}

A $250 \mathrm{~mm} \mathrm{f} / 4$ reflector with a flat secondary was also built, but it suffered from nonrepeatable pointing variations at the arcsecond level, so we abandoned the design as a viable option for CCAT. This experience illustrates the main difficulty with reflecting pointing telescopes-heavy mirrors are difficult to mount when repeatability is a driving requirement.

\section{CONCLUSIONS}

We have built and tested a pair of refractor pointing telescopes that achieve an rms differential pointing stability of $0.1^{\prime \prime} \mathrm{hr}^{-1}$ over $2 \mathrm{hr}, 0.05^{\prime \prime}$ day $^{-1}$ over 3 days, $0.03^{\prime \prime} \mathrm{K}^{-1}$, and $0.1^{\prime \prime}$ after a $90^{\circ}$ change in elevation.

The telescopes have a $100 \mathrm{~mm} / 15$ achromat mounted in a rolled Invar tube. The objective is glued to three blade flexures that are attached to the inside of the tube, and the camera is mounted in the center of a circular plate at the end of the tube. Fans continuously blow air through the tube, which is insulated inside and out. All the metal parts are made of Invar, and the tube is mounted on blade flexures.

CCAT will be equipped with two or three of these small telescopes for measuring pointing model terms and pointing stability.

\section{REFERENCES}

Carlstrom, J. E., et al. 2011, PASP, 123, 568

$\rightarrow$ Edlen, B. 1966, Metrologia, 2, 71

Leighton, R. B. 1978, Final Technical Report for NSF Grant 73-04908 (Pasadena: California Inst. Technology)

Mangum, J. G. 2000, ALMA Memo 288, An Optical Pointing System for the ALMA Prototype Antennas (Charlottesville: NRAO)
This work was supported by the John B. and Nelly Kilroy Foundation.

\footnotetext{
$\rightarrow$ Mangum, J. G., Baars, J. W. M., Greve, A., Lucas, R., Snel, R. C., Wallace, P., \& Holdaway, M. 2006, PASP, 118, 1257

Ukita, N., Ikenoue, B., \& Saito, M. 2008, Publ. Natl. Astron. Obs. Japan, 11, 1

Yoder, P. R., Jr. 2006, Opto-Mechanical Systems Design (3rd ed.; Boca Raton: CRC Press), chap. 8
} 